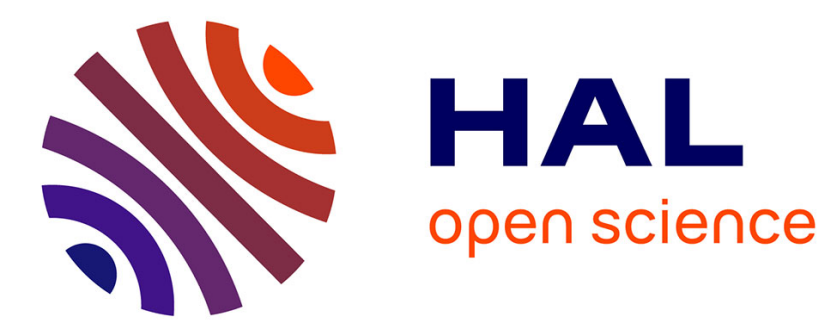

\title{
Les Camélidés sud-américains : Le point des connaissances
}

\author{
M. Prud'Hon, R. Cordesse, S. de Rouville, J. Thimonier
}

\section{To cite this version:}

M. Prud'Hon, R. Cordesse, S. de Rouville, J. Thimonier. Les Camélidés sud-américains : Le point des connaissances. Productions Animales, 1993, 6 (1), pp.5-15. hal-00896037

\section{HAL Id: hal-00896037 https://hal.science/hal-00896037}

Submitted on 1 Jan 1993

HAL is a multi-disciplinary open access archive for the deposit and dissemination of scientific research documents, whether they are published or not. The documents may come from teaching and research institutions in France or abroad, or from public or private research centers.
L'archive ouverte pluridisciplinaire HAL, est destinée au dépôt et à la diffusion de documents scientifiques de niveau recherche, publiés ou non, émanant des établissements d'enseignement et de recherche français ou étrangers, des laboratoires publics ou privés. 
INRA Prod. Anim., $1993,6(1), 5-15$.

\section{PRUD'HON, R. CORDESSE,} S. de ROUVILLE, J. THIMONIER,

INRA-ENSA, Unité de Zootechnie Méditerranéenne, 2 Place Viala, 34060 Montpellier Cedex

\section{Les Camélidés sud-américains : Le point des connaissances}

Depuis l'époque de Buffon où ils ont été introduits en France, les camélidés sud-américains sont restés cantonnés, le plus souvent, dans les parcs zoologiques. Toutefois, depuis dix ans, des éleveurs, d'abord isolés puis groupés pour la plupart dans l'association Alpagas et Lamas de France qui compte une cinquantaine de membres, ont mis en place des élevages de camélidés sud-américains. Les effectifs sont modestes (moins de 1000 têtes réparties de façon égale entre alpagas et lamas) mais l'intérêt suscité par cet élevage est important. Nombreux sont les éleveurs souhaitant acquérir des alpagas si les conditions d'importation le permettent. Les demandes d'information se multiplient ; une première journée technique, organisée par l'association Alpagas et Lamas de France, s'est tenue à Lyon en septembre 1992. L'Unité INRA de Zootechnie méditerranéenne de Montpellier a cherché dès 1988 à mieux connaitre les caractéristiques de ces camélidés en prenant pour modèle le Lama. Cet article fait le point des connaissances principales actuelles.

\section{Résumé}

L'élevage de camélidés sud-américains a pu être envisagé dans le cadre d'une politique d'entretien de l'espace et de diversification des productions. Cette étude bibliographique permet de mieux évaluer les possibilités et les limites de ces espèces.

Il existe quatre espèces de camélidés sud-américains, deux sauvages, le Guanaco et la Vigogne, et deux domestiques, l'Alpaga et le Lama. Elles vivent sur les hauts plateaux andins.

Alpagas et lamas peuvent se reproduire en toutes saisons. La reproduction présente des traits originaux : les mâles, pubères à un an, sont handicapés jusqu'à deux ans par des adhérences préputiales et sont rarement exploités avant trois ans. Chez la femelle, l'ovulation, simple le plus souvent, est provoquée par l'accouplement. Un seul foetus se développe, toujours dans la corne gauche de l'utérus. La gestation dure 345 jours environ. Le réaccouplement est possible peu après la parturition. Les jeunes pèsent $7-8 \mathrm{~kg}$ (alpagas) à $10-11 \mathrm{~kg}$ (lamas) à la naissance. Le sevrage a lieu vers 7 à 9 mois.

Alpagas et lamas sont des ruminants, caractérisés par un estomac à 3 compartiments, sans homologie avec les quatre compartiments de l'estomac des ovins, bovins et caprins. Leur contenu, très sec, est riche en bactéries.

Les alpagas consomment essentiellement de l'herbe, même en touffes hautes et grossières ; ce sont des "pâtureurs". Les lamas, normalement "pâtureurs", consomment aussi, de façon minoritaire, des espèces arbustives si l'herbe se fait rare. Alpagas et lamas mangent généralement moins que les ovins et caprins $\left(30\right.$ à $55 \mathrm{~g} \mathrm{MS} / \mathrm{kg} \mathrm{P}^{0,75}$ selon les fourrages, soit 20 à $40 \%$ de moins à régime comparable). L'utilisation digestive des matières organiques et azotées est égale (fourrages de bonne qualité) ou supérieure (fourrages grossiers, pauvres en protéines) à celle de ces deux espèces.

Les camélidés sud-américains coexistent sans problème avec les petits ruminants ; leur organisation sociale (hiérarchie, protection du troupeau) nécessite un minimum de précautions dans la conduite des animaux.

La principale production des alpagas est leur toison formée de fibres fines ( 25 à $30 \mu$ ) et médullées, d'un rendement élevé ( $80 \%$ ) mais d'un poids modeste (1,7 et $2,8 \mathrm{~kg}$ chez les femelles et les mâles). Les lamas ont des toisons plus grossières comportant également des poils. Elles sont utilisables en artisanat.

En France, il n'existe encore aucune étude économique sérieuse qui permette d'inciter les éleveurs à investir dans l'élevage de camélidés sud. américains. 
Dans un contexte de productions animales excédentaires, il est de plus en plus difficile d'assurer la survie des élevages dans les régions à fortes contraintes climatiques, notamment dans les régions méditerranéennes et de montagne. Un certain nombre d'éleveurs, isolés ou associés, explorent les possibilités offertes par des espèces peu exploitées jusqu'alors : cerfs, bisons d'Europe ou d'Amérique, autruches, chèvres mohair, camélidés sud-américains, etc...

Face à ces initiatives, isolées mais souvent spectaculaires, la grande presse et les médias font chorus, avant même que ne soient connues les caractéristiques et les aptitudes de ces "nouvelles espèces".

Il a donc semblé intéressant de faire le point sur les camélidés sud-américains dont l'élevage démarre à peine en France, mais suscite des espérances tant pour la production de fibres textiles de qualité que pour l'entretien de l'espace et le tourisme vert.

\section{1 / Principales espèces, caractéristiques et distribution}

Il existe quatre espèces de camélidés sudaméricains, deux espèces sauvages, le Guanaco et la Vigogne, et deux espèces domestiques, le Lama et l'Alpaga (tableau 1). Très proches les unes des autres sur le plan génétique $(2 \mathrm{n}=74$ chromosomes), ces espèces sont inter-fertiles ; les hybrides les plus fréquents sont le Huarizo (Lama x Alpaga), sans intérêt zootechnique, et le Paco-Vicuna (Alpaga x Vigogne), intéressant. pour sa laine. L'hypothèse selon laquelle le Lama et l'Alpaga seraient les formes domestiquées respectivement du Guanaco et de la Vigogne est partiellement infirmée par les études de biologie moléculaire (Vidal-Rioja et al 1991) qui montrent la très nette originalité de la Vigogne, l'existence d'un groupement monophylétique composé du Guanaco et du Lama ; l'Alpaga se différencie de ceux-ci uniquement au niveau des caractéristiques des ADN satellites isolés par les enzymes de restriction.

La famille des camélidés est apparue en Amérique du Nord à l'Eocène supérieur puis a migré vers l'Asie et l'Amérique du Sud. D’abord chassés, les camélidés sud-américains ont été domestiqués 6000 à 3000 ans avant J.C.. Ils ont joué et jouent encore un rôle essentiel dans l'économie des hauts plateaux andins (vêtements, viande, transport). L'espèce domestique la plus intéressante en raison de la qualité de ses fibres est l'Alpaga dont il existe deux races se distinguant par la nature de leur toison, les Huacaya et les Suri. Les alpagas vivent dans les zones d'altitude élevée, 4200 à 4800 mètres, dans les Andes péruviennes et boliviennes et, à un moindre degré, sur les plateaux chilien et argentin.

Les lamas, dont il existe également deux races, les Tapada et les Ccara Sullo, sont des animaux de bât. Plus lourds et réputés plus rustiques que les alpagas, ils colonisent cependant les zones d'altitude plus faible (2 300 à $4000 \mathrm{~m}$ ) de ces mêmes pays, coexistant avec de grands troupeaux ovins. Leur élevage décline avec la mécanisation des transports.

La Vigogne habite les régions de très haute altitude $(4800$ à $5000 \mathrm{~m})$ des Andes péruviennes, avec quelques îlots en Argentine, en Bolivie et au Chili. Elle est le plus souvent cantonnée dans des réserves.

La Vigogne est légère, parfaitement adaptée à la vie à très haute altitude et à la raréfaction de l'oxygène. Elle est d'une grande vivacité et porte la laine la plus fine de tous les animaux (Fowler 1989).

Tableau 1. Classification et caractéristiques des camélidés sud-américains (d'après Calle Escobar 1982).

\begin{tabular}{|c|c|c|c|c|}
\hline $\begin{array}{l}\text { Classification } \\
\text { Classe : Mammiferes, } \\
\qquad \text { ordre : Arti }\end{array}$ & $\begin{array}{l}\text { uminants, } \\
\text { upe : Tylopod } \\
\text { Famil }\end{array}$ & élidés. & & \\
\hline Genre & & Lama & & Vicugna \\
\hline $\begin{array}{l}\text { Espèce } \\
\text { Nom courant }\end{array}$ & $\begin{array}{l}\text { L. GLAMA } \\
\text { Lama }\end{array}$ & $\begin{array}{l}\text { L. PACOS } \\
\text { Alpaga }\end{array}$ & $\begin{array}{c}\text { L.GUANICOE } \\
\text { Guanaco }\end{array}$ & $\begin{array}{l}\text { V. VICUGNA } \\
\text { Vigogne }\end{array}$ \\
\hline Races & $\begin{array}{c}\text { Ccara Sullo } \\
\text { Tapada }\end{array}$ & $\begin{array}{c}\text { Huacaya } \\
\text { Suri }\end{array}$ & $\begin{array}{c}\text { Cacsilensis } \\
\text { Bermejo }\end{array}$ & \\
\hline Effectifs & $4 \times 10^{6} ?$ & $3 \times 10^{6}$ & $10^{5} ?$ & $6,5 \times 10^{!}$ \\
\hline Poids vif $(\mathrm{kg})$ & $90-110$ & $75-85$ & $80-90$ & $35-50$ \\
\hline Hauteur au garrot $(\mathrm{cm})$ & $100-125$ & $85-95$ & $100-120$ & $70 \cdot 90$ \\
\hline Poids de toison (kg) & $1,5-3,5$ & $1,5-2,0$ & $1,0-2,0$ & $0,2-0,3$ \\
\hline Longueur des mèches $(\mathrm{cm})$ & $25-30$ & $10-30$ & $10-15$ & \\
\hline Finesse $(\mu)$ & $25-80$ & $15-30$ & $40-80$ & $6-10$ \\
\hline
\end{tabular}


Le Guanaco, plus lourd, est plus ubiquiste ; il est présent du niveau de la mer jusqu'à des altitudes élevées et de l'équateur à la Terre de Feu. Le plus gros noyau se situe en Patagonie. Il est capable de vivre en forêt.

La diffusion des camélidés sud-américains hors de l'Amérique du Sud est restée très limitée en raison d'une interdiction d'exporter mise en place vers le milieu du dix-neuvième siècle (Fowler 1989). Des essais d'introduction d'alpagas en France et en Espagne (18ème siècle) puis en Australie (milieu du 19ème siècle) semblent avoir été des échecs (Calle Escobar 1982). Depuis 1980, un certain nombre d'exportations d'alpagas et de lamas ont été pratiquées, via le Chili, vers les Etats Unis où l'effectif est d'environ 15000 alpagas, l'Australie, la Nouvelle-Zélande, etc. En France, les introductions sont restées très limitées et la majeure partie des études ont porté sur des animaux provenant de parcs zoologiques.

La plupart des données scientifiques concernant les camélidés sud-américains proviennent de centres de recherches sud-américains, notamment l'IVITA (Instituto Veterinario de Investigaciones Tropicales y de Altura) qui dépend de l'Université Nationale Majeure de San Marcos à Lima, et l'Université Nationale Technique de Puno. Plus de la moitié des travaux concernent les alpagas.

Récemment toutefois, des études ont été publiées aux USA (universités du Texas et de Californie), en Nouvelle-Zélande, en Ecosse, etc. En France, plusieurs documents de synthèse (Avila Cazorla 1986, Segrétain 1987, Brunschwig 1988, Giudicelli 1991, Bravo 1992) et des premiers résultats expérimentaux, obtenus notamment à l'Unité de Zootechnie Méditerranéenne (INRA-ENSA) de Montpellier, constituent des repères utiles.

\section{2 / Reproduction}

Dans les conditions d'élevage des hauts plateaux andins, la période de reproduction se situe en été, de décembre à mars, à la saison des pluies qui est aussi celle des disponibilités fourragères maximales, mais, selon Sumar et Garcia (1986), la reproduction est possible tout au long de l'année chez les animaux bien nourris. En fait, il semble qu'il existe malgré tout une certaine saisonnalité. En effet, des alpagas transférés en Nouvelle-Zélande $\left(45^{\circ}\right.$ de latitude sud), élevés sur prairies irriguées, ont eu des taux de fertilité de $22 \%$ et $66 \%$ respectivement au printemps et à l'automne (Davis et al 1991). Au Pérou, le pourcentage de femelles ovulant et le taux de fécondation ont un maximum en jours courts chez les alpagas primipares et multipares. Toutefois, la sécheresse et le déficit alimentaire abaissent ces taux au mois d'aôut. Dans la pratique, la durée de gestation supérieure à 11 mois et le réaccouplement peu après la parturition aboutissent à une grande stabilité de l'époque de reproduction.
Comme dans la plupart des espèces, la puberté dépend du développement corporel. Selon Sumar et Garcia (1986), un alpaga mâle d'un an pesant $35 \mathrm{~kg}$ manifeste déjà de l'intérêt pour les femelles. Toutefois, $92 \%$ des mâles d'un an et $30 \%$ des mâles de deux ans sont handicapés par des adhérences préputiales, caractéristiques de l'immaturité sexuelle, qui disparaissent avec la secrétion de testostérone (Fernandez-Baca 1970). Au Pérou, l'âge classique de mise à la reproduction des mâles alpagas est de trois ans; ils pèsent alors $65 \mathrm{~kg}$, ont des testicules d'environ $20 \mathrm{~g}$ et n'ont plus d'adhérences ce qui permet l'érection du pénis (les camélidés ont un $\mathrm{S}$ pénien comme les taureaux).

Chez les femelles, la puberté est atteinte lorsque leur poids est d'environ 35 à $40 \mathrm{~kg}$, soit $50 \%$ à $60 \%$ du poids adulte. Ce poids est atteint dès l'âge de six mois si les conditions sont très favorables (Davis et al 1991, en Nouvelle-Zélande), mais le plus souvent vers treize mois et jusqu'à deux ans dans les Andes où 55 à $60 \%$ des premières mise bas surviennent à 3 ans.

A la puberté, les ovaires ont une surface d'aspect granuleux avec des follicules de plus de $5 \mathrm{~mm}$ de diamètre. Plusieurs follicules se développent simultanément, par "vagues" successives, indépendamment, le cas échéant, de la présence de corps jaunes sur l'ovaire du côté opposé (Bravo et al 1990, Adam et al 1992).

L'ovulation est normalement induite par l'accouplement effectif ou par injection intraveineuse d'hCG mais non par de simples chevauchements sans intromission du pénis. Elle survient environ 26 heures après le coït, parfois avec retard et au delà de 30 heures dans $38 \%$ des cas selon Sumar et Garcia (1986). Les ovulations multiples sont rares : 2 à $8 \%$ selon les auteurs (San Martin et al 1968, Sumar et Garcia 1986)

Classiquement, chez les camélidés sud-américains, les mâles sont élevés séparément des femelles. A la mise en lutte, l'activité sexuelle des mâles et des femelles est intense au cours des premiers jours. Poursuites et accouplements se multiplient; les femelles réceptives, c'est-à-dire celles qui acceptent l'accouplement, adoptent plus ou moins vite la position assise (baraquée); certaines femelles s'installent même dans cette position à proximité immédiate d'un mâle en cours d'accouplement. Le coït, parfois interrompu et repris, dure de 5 à 50 minutes. La durée moyenne est plus longue lorsqu'un seul mâle est avec un lot de femelles (20 minutes pour un mâle avec 20 femelles contre 8 minutes pour 5 mâles avec 100 femelles selon Calle Escobar 1982). Pollard et al (1991), avec des alpagas luttant en boxes deux jours consécutifs, enregistrent une durée moyenne de 15,5 minutes ; la durée est supérieure de 2 minutes le premier jour de lutte comparé au second. En dehors des records (18 accouplements en 24 heures), l'activité sexuelle des mâles est très intense les deux premiers jours de lutte : 6,3 poursuites et 5,5 accouplements (Pollard et al 1991). Environ 70 à $80 \%$ des femelles réceptives sont effectivement
Lamas et alpagas peuvent se reproduire toute l'année. Le poids à la naissance est de 7 à $8 \mathrm{~kg}$ pour l'alpaga et de 10 à $11 \mathrm{~kg}$ pour le lama. 
Tableau 2. Relations entre la localisation des corps jaunes et celle des embryons (d'après Sumar et Garcia 1986).

\begin{tabular}{|lcc|cccc|}
\hline Localisation & & & \multicolumn{4}{|c|}{ Localisation des embryons } \\
des corps jaunes & nombre & $\%$ & Corne droite & \multicolumn{2}{c|}{ Corne gauche } \\
& & & nb & $\%$ & nb & $\%$ \\
\hline Ovaire droit & 477 & 50,9 & 12 & 2,5 & 465 & 97,5 \\
Ovaire gauche & 440 & 47,4 & 3 & 0,7 & 437 & 99,3 \\
Deux ovaires & 16 & 1,7 & 0 & 0 & 16 & 100,0 \\
Total & 933 & 100 & & & & \\
\hline
\end{tabular}

saillies en une semaine. Cette activité retombe vite mais elle retrouve son niveau initial lorsque les partenaires sont renouvelés ou intervertis.

Environ $80 \%$ des femelles saillies ovulent, ce poucentage variant avec la saison. L'ovulation a lieu avec la même fréquence sur les ovaires droit ou gauche (tableau 2). Le corps jaune se développe pour atteindre un diamètre maximum, d'environ $12 \mathrm{~mm}, 6$ jours après l'ovulation, puis régresser les 6 jours suivants si la fécondation n'a pas eu lieu ; il n'y aurait pas de pseudo-gestation (Adams et al 1991). Si la fécondation a lieu, le corps jaune poursuit son développement, atteint son diamètre maximum 21 jours environ après l'ovulation puis persiste jusqu'à la parturition; il est indispensable tout au long de la gestation. Le taux de progestérone plasmatique est étroitement corrélé avec le diamètre du corps jaune $(r=0,83$, Adams et al 1991).

Les données sur le volume, la concentration et la qualité du sperme d'Alpaga et de Lama sont rares car la collecte en est difficile en raison de la durée et de la position lors de l'accouplement. Par électro-éjaculation, FernandezBaca et Calderon (1966), cités par Sumar et Garcia (1986), recueillent des éjaculats contenant, au mieux, $255 \times 10^{6} \mathrm{spz} / \mathrm{ml}$. Au vagin artificiel, Sumar et Leyva (1981), cités par Sumar et Garcia (1986), collectent des éjaculats de $12,5 \mathrm{ml}$ et $600 \times 10^{6} \mathrm{spz} / \mathrm{ml}$. Ce sperme est très visqueux et serait éjaculé de façon continue au cours du coit.

La fertilité dépend probablement beaucoup de la qualité des éjaculats. La pratique du renouvellement fréquent des mâles a permis de l'améliorer sensiblement $(81 \%$ vs 53 à $61 \%$ ) dans les conditions d'élevage du Pérou.

La nidation est tardive, 30 jours, et le développement foetal a presque toujours lieu dans la corne utérine gauche, quel que soit le côté d'ovulation (tableau 2). Ceci suppose un mécanisme de migration mal connu.

Le diagnostic de gravidité peut être réalisé par dosage radio-immunologique de la progestérone sanguine ou de stéroïdes urinaires (Bravo et al 1991), par présentation à des mâles vasectomisés, ou, au delà de 45 jours, par palpation rectale ou par échographie (Alarcon et al 1990).

Dans les conditions d'élevage du Pérou, la mortalité embryonnaire paraît importante : Fernandez-Baca et al (1970) ont trouvé, par abattages, deux fois moins de femelles gravides à 30 jours que 3 jours après accouplement ( $35 \%$ de gravides vs $70 \%$ ).

Le placenta est diffus et de type épithéliochorial.

La durée de gestation est d'environ 335 jours chez les lamas, 342 à 345 jours chez les alpagas. A l'approche de la mise bas, les femelles s'isolent du troupeau et présentent des signes de nervosité (posture des oreilles, alternance des positions debout et couchée...). La parturition a lieu de jour, par beau temps, et dure peu (5 à 90 minutes). Environ $67 \%$ (Davis et al 1991) à 93,5 \% (Sumar et Garcia 1986) des naissances surviennent entre 7 et 13 heures. Après la parturition, l'involution de l'utérus est très rapide, environ 5 jours.

Après la mise bas les mères flairent leurs petits mais ne les lèchent pas. Elles pratiquent facilement l'adoption. Très rapidement les nouveau-nés se lèvent et se mettent à téter. La production de lait d'alpaga et de lama est mal connue car les tétines sont très courtes et difficiles à traire ; elle serait de l'ordre de 200 à $500 \mathrm{ml}$ par jour (Moro 1956, cité par Avila Cazorla 1986). Selon Fowler (1989), les laits d'alpaga et de lama sont très comparables - eau : 86,2 \% ; matières grasses : $5,66 \%$; protéines : $4,25 \%$; lactose : $3,34 \%$ - de composition intermédiaire entre celles du lait de vache et de brebis. Le sevrage des jeunes a lieu vers l'âge de 7 à 9 mois.

Enfin, les techniques modernes de reproduction, insémination artificielle et transfert d'embryons, ont été mises au point. L'IA est de mise en oeuvre difficile : collecte de semence longue et délicate, induction de l'ovulation par des mâles vasectomisés ou des traitements hormonaux (750 UI d'hCG), utilisation de sperme frais par voie intra-cervicale 35 à 45 heures après cette induction ; de ce fait, elle demeure du domaine du laboratoire, éventuellement pour réaliser des hybridations interspécifiques.

La superovulation et le transfert d'embryons ont été mis au point récemment (Palomino et al 1985, Bourke et al 1992) ; ceci requiert la synchronisation et l'induction des ovulations chez les donneuses et les receveuses, la superovulation (1000 UI de PMSG) et la fécondation des donneuses, la collecte des embryons par voie trans-cervicale, 8 à 14 jours plus tard, et leur mise en place chez les receveuses synchrones. C'est également une technique de laboratoire. 


\section{3 / Comportement alimentaire et nutrition}

Les camélidés sont des ruminants ; ils ont la faculté de régurgiter des bols alimentaires et de les mastiquer à nouveau. Comparés aux ruminants "classiques" (bovins, ovins, caprins), ils se distinguent, sur le plan anatomique, par :

- des lèvres supérieures fendues, chaque moitié étant mobile et indépendante, permettant une palpation sélective ;

- un estomac à 3 compartiments appelés $C_{1}$ ( $80 \%$ du volume total), $\mathrm{C}_{2}$ et $\mathrm{C}_{3}$. Les deux premiers ont un rôle essentiellement fermentaire grâce à la présence d'une flore et d'une faune anaérobies. $\mathrm{C}_{1}$ et $\mathrm{C}_{2}$ ont une muqueuse riche en nodules de cellules sécrétrices (mucus) qui recouvrent les parties ventrale et latérales du compartiment; la tunique musculaire est animée de puissantes ondes de contraction allant du sac caudal au sac crânial ; le contenu de $C_{1}$ et $\mathrm{C}_{2}$ est beaucoup plus sec que celui du rumen des ovins (Fowler 1989). En dépit d'une certaine analogie fonctionnelle, il n'existe pas d'homologie structurelle entre les compartiments $\mathrm{C}_{1}, \mathrm{C}_{2}$ et $\mathrm{C}_{3}$ et l'un des quatre compartiments (rumen, réseau, feuillet, caillette) de l'estomac des ruminants classiques. Par sa fonction, $\mathrm{C}_{3}$ joue un rôle analogue à la caillette.

- une gouttière oesophagienne très peu marquée.

Les études concernant les rythmes d'activité, la nature quantitative et qualitative des régimes ingérés, leur digestibilité et leur dégradabilité, sont assez nombreuses mais souvent peu diffusées (mémoires, thèses, colloques) et difficilement comparables compte tenu des sites expérimentaux, des méthodologies utilisées et des espèces comparées. Un certain nombre de points peuvent, malgré tout, être soulignés.

\section{Rythme d'activité}

Lamas et alpagas ont une activité d'ingestion diurne d'une durée moyenne d'environ 8-9 heures, variant en fonction des ressources disponibles : 7 h 30 pour des lamas sur prairie irriguée de Crau (Segrétain et al 1989), 12 h 30 sur des parcours de garrigue en fin d'exploitation (de Rouville et al 1991). Dumont (1991) n'a pas constaté d'interruption marquée de pâturage au cours des heures chaudes de l'été comme c'était le cas chez des chèvres du Rove, ni de synchronisation des périodes d'ingestion au sein du troupeau.

La rumination est essentiellement nocturne ; elle durerait environ 6 heures ; elle n'excède pas $3 \%$ de la période diurne contre $73 \%$ pour l'ingestion et $24 \%$ pour le repos et les déplacements (de Rouville et al 1991). La rumination a lieu normalement en position couchée.
L'ingestion de ligneux augmente avec le temps de séjour sur les parcelles, mais, comparés aux chèvres, les lamas sont de piètres débroussailleurs.

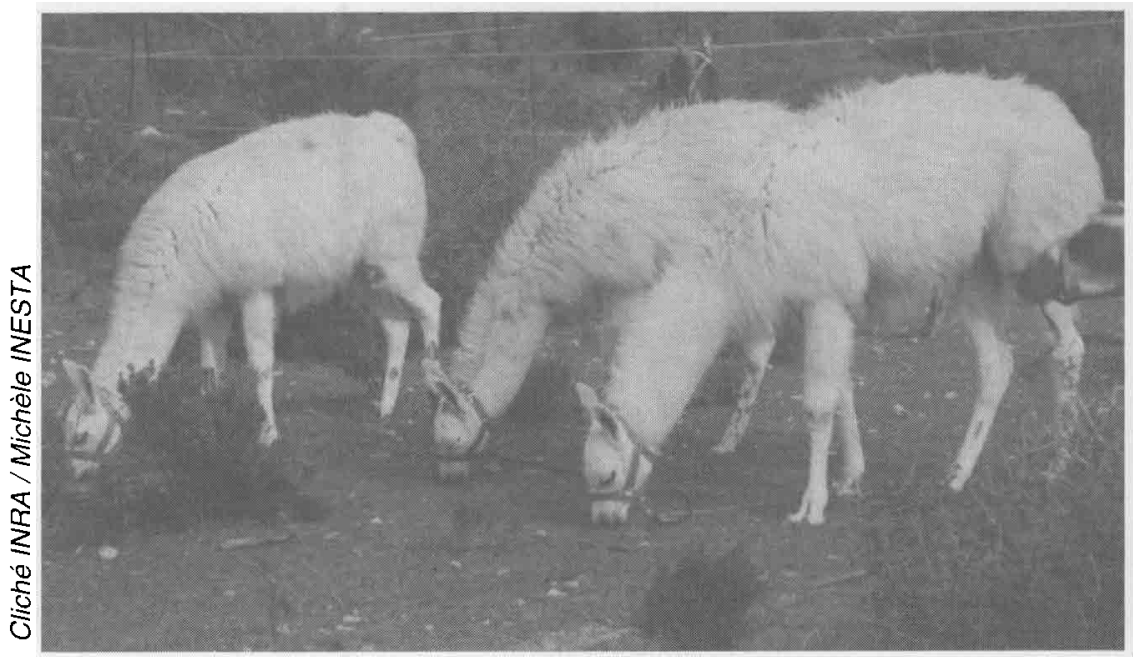

\section{Choix alimentaires}

Divers modes d'étude ont été utilisés pour apprécier, à diverses saisons, les choix alimentaires des lamas ou des alpagas : observations visuelles (de Rouville et al 1991), coups de dents stratifiés (Dumont 1991, Villca et al 1992), analyse micro-histologique des fèces (Bryant et Farfan 1984), prélèvements de bols par des canules oesophagiennes (Reiner et Bryant 1986).

Outre des indications botaniques sur les espèces ingérées dont l'intérêt est limité au site expérimental, ces études montrent que les alpagas sont essentiellement des "pâtureurs", c'est-à-dire des mangeurs d'herbe, consommant presque exclusivement des graminées et autres herbacées, notamment des carex dans des zones humides, alors que les lamas sont également capables de consommer des ligneux. Avila Cazorla (1986), citant Casamitjana (1976), souligne toutefois que, parmi les graminées, les alpagas ont une prédilection pour les touffes d'herbe haute et dure : Festuca dolicophylla, Dristichia muscoides, Scirpus rigidus, Junco ondeyanus, etc.

Sur garrigues méditerranéennes riches en chênes kermès, genévriers et genêts scorpions, le temps consacré à l'ingestion de ligneux et les proportions consommées augmentent avec le temps de séjour sur les parcelles (de Rouville et al 1991, Dumont 1991) ; cependant, comparés aux chèvres, les lamas sont de piètres "débroussailleurs" : selon Dumont (1991), les chèvres n'ont jamais consommé plus de $35 \%$ de MS d'herbacées alors que c'est le seuil minimum observé pour des lamas sur des parcours déjà très exploités.

Le choix alimentaire varie avec la saison en fonction notamment de facteurs phénologiques : consommation de genêt scorpion au moment de la floraison (de Rouville et al 1991), de graines au cours de la période sèche (Bryant et Farfan 1984); cependant l'effet saisonnier joue surtout au travers des effets de la sécheresse sur la composition des végétaux disponibles (San Martin 1991).

\section{Les camélidés sont des ruminants mais avec un estomac à 3 compartiments dont les 2 premiers ont un contenu beaucoup plus sec que celui du rumen des ovins.}


Tableau 3. Quantités ingérées (en g MS/kg $P^{0,75}$, sauf * : matière organique ingérée) par différents camélidés sud-américains : Alpaga (A) et Lama (L).

\begin{tabular}{|c|c|c|c|c|}
\hline Référence & Espèce & Régime & \multicolumn{2}{|c|}{ Quantités Ingérées } \\
\hline $\begin{array}{l}\text { Cordesse et al } 1992 \\
\text { Reiner et al } 1987\end{array}$ & $\begin{array}{l}\text { L } \\
L \\
L \\
L \\
A \\
A\end{array}$ & $\begin{array}{c}\text { Foin luzerne } \\
\text { Paille blé + NH3 } \\
\text { Paille blé + urée } \\
\text { Paille blé } \\
\text { Foin d'avoine } \\
\text { Foin de ray-grass }\end{array}$ & \multicolumn{2}{|c|}{$\begin{array}{l}58,9 \\
49,2 \\
41,9 \\
36,5 \\
31,6^{*} \\
28,8^{*}\end{array}$} \\
\hline $\begin{array}{l}\text { Dumont } 1991 \\
\text { Huisa } 1986 \\
\text { San Martin } 1987\end{array}$ & $\begin{array}{l}\text { L } \\
\text { L } \\
\text { A } \\
\text { L } \\
\text { A } \\
\text { L } \\
\text { A } \\
\text { L }\end{array}$ & $\begin{array}{c}\text { Garrigue, br, qc } \\
\text { Prairies andines } \\
\text { fd, pt } \\
\text { fd } \\
\text { fd } \\
\text { fr } \\
\text { fr } \\
\text { lp, fru, tr } \\
\text { lp, fru, tr } \\
\text { fo }\end{array}$ & $\begin{array}{c}\text { sais. sèche } \\
42,4 \\
40,3 \\
35,8 \\
44,3 \\
42,4 \\
67,0 \\
61,8 \\
34,2\end{array}$ & $\begin{array}{c}\text { sais. humide } \\
41,2 \\
38,6 \\
54,0 \\
39,7 \\
41,2 \\
51,2 \\
53,3\end{array}$ \\
\hline
\end{tabular}

br: Brachypodium ramosum ; fd : Festuca dolicophylla ; fo : Festuca orthophylla ; fr : Festuca rigida ; fru : Festuca rubra ; lp : Lolium perenne; $\mathrm{mf}$ : Muhlenbergia fastigiata ; $\mathrm{pt}$ : Plantago tubulosa ; qc : Quercus coccifera ; tr : Trifolium repens.

Tableau 4. Digestibilité comparée de différents fourrages par des lamas, des ovins, et des caprins.

\begin{tabular}{|c|c|c|c|c|c|}
\hline \multirow[t]{2}{*}{ Référence } & \multirow[t]{2}{*}{ Fourrage } & \multicolumn{2}{|c|}{ dMO } & \multicolumn{2}{|c|}{ dMA } \\
\hline & & Lama & Caprin & Lama & Caprin \\
\hline \multirow[t]{5}{*}{ Cordesse et al 1992 (France) } & Foin de luzerne & 61,0 & 61,2 & 71,9 & 72,1 \\
\hline & Paille $\mathrm{NH}_{3}$ & 60,5 & 59,0 & 43,6 & 42,6 \\
\hline & Paille + Urée & 58,9 & & 54,9 & \\
\hline & Paille & 55,0 & & $\varepsilon$ & \\
\hline & & Lama & Ovin & Lama & Ovin \\
\hline Villca et al (Pérou) & Paja brava & 56,9 & 44,1 & 59,0 & 42,5 \\
\hline
\end{tabular}

dMO : digestibilité de la matière organique ; $\mathrm{dMA}$ : digestibilité des matières azotées.

\section{Rapportés au poids métabolique, les niveaux d'ingestion sont plus faibles chez les camélidés que chez les ovins et caprins, de $20 \grave{a} 40 \%$ selon le régime.}

La comparaison des indices de similarité entre la composition botanique des végétations disponibles et ingérées par des alpagas, lamas et moutons met en évidence la moindre sélectivité des lamas par rapport aux alpagas et surtout aux moutons, en saison sèche comme en saison humide (San Martin 1991).

\section{Niveau d'ingestion}

Le niveau d'ingestion de matière sèche de la ration est évidemment très variable selon les ressources disponibles (tableau 3 ). Il évolue par exemple de 36,5 à $58,9 \mathrm{~g} \mathrm{MS} / \mathrm{kg} \mathrm{P}^{0,75}$ pour des lamas recevant de la paille de blé ou du foin de luzerne (Cordesse et al 1992), de 26 à $53 \mathrm{~g} / \mathrm{kg} \mathrm{P}^{0,75}$ pour des lamas utilisant en été des parcelles de garrigue respectivement "usées" ou nouvelles (Dumont 1991).

Dans toutes les études comparatives, les camélidés sud-américains ont des niveaux de consommation, rapportés au poids métabolique, sensiblement inférieurs à ceux des ovins et des caprins. San Martin (1991) relève des écarts de 20 à $26 \%$ entre alpagas et ovins, de 30 à $39 \%$ entre ovins et lamas au Pérou. En France, Dumont (1991) note une différence de $28 \%$ entre lamas et chèvres en garrigue. Soulignons toutefois que certains fourrages très grossiers et très pauvres en protéines sont consommés par les lamas mais non par les ovins (Cordesse et al 1992).

\section{Digestibilité et dégradabilité des rations}

Les études comparatives de digestibilité des fourrages par les camélidés sud-américains et les ovins (San Martin 1991, Cordesse et al 1992, Villca et al 1992) permettent de penser qu'il n'existe pas de différences de digestibilité des matières organique et azotée lorsque les fourrages sont de bonne qualité. En revanche, la digestibilité des fourrages grossiers et pauvres serait nettement supérieure chez les camélidés. Dans une même espèce, la digestibilité est fonction de la qualité du fourrage (tableau 4). Dans une comparaison entre alpagas et ovins recevant des régimes de différents niveaux protéiques ou de cellulose, San Martin (1991) relève des digestibilités de la matière sèche allant de 51 à $73 \%$ (lamas) et 41 à $75 \%$ (moutons) avec des régimes variant de 7 à $11 \%$ de matières azotées totales. La digestibilité est peu différente entre espèces pour des régimes dont les teneurs en NDF (neutral detergent fiber) varient de 68 à $42 \%$; la digestibilité de la matière sèche varie respectivement de 58 à $67 \%$ chez les lamas et de 52 à $65 \%$ chez les moutons. 
Les études concernant la dégradabilité comparée d'un même fourrage (paille traitée à l'ammoniac) par des lamas et des chèvres élevés sur parcours de garrigue à différentes saisons (Cordesse 1992) montrent que l'activité cellulolytique est très voisine chez les deux espèces. La concentration des acides gras volatils (AGV), témoin de la dégradation de la matière organique donc de l'importance de l'activité bactérienne, semble légèrement plus élevée chez le lama.

Les besoins énergétiques, azotés et minéraux d'entretien et de production sont très mal connus. Selon Carmean et al (1992), les besoins énergétiques de lamas à jeun ou à l'entretien seraient respectivement 59,3 et

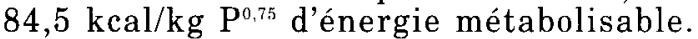
Engelhardt et Schneider (1977), étudiant trois lots de lamas à l'entretien, recevant respectivement une alimentation ad libitum, à $80 \%$ ou à $60 \%$ de ce niveau, enregistrent des consommations d'énergie métabolisable de $66,7,60,8$ et $56,1 \mathrm{kcal} / \mathrm{kg} \mathrm{P}^{0,75}$. Pour les besoins azotés, les données sont encore moins nombreuses. Les besoins des camélidés sud-américains seraient plus faibles que ceux des ovins en raison de leur aptitude à recycler l'urée et à l'utiliser pour la synthèse protéique (San Martin 1991).

Enfin, les besoins en eau, rapportés à la matière sèche ingérée, seraient plus réduits chez les lamas que chez les alpagas et les ovins $(1,6 \mathrm{~kg} / \mathrm{kg}$ MS chez les lamas vs 2,2 chez les ovins).

\section{4 / Production et pathologie}

Traditionnellement, les camélidés sud-américains sont exploités pour le transport, la production de viande et de fibres textiles. Seuls les lamas sont bâtés. Selon Calle Escobar (1982), un lama adulte, qui pèse environ $100 \mathrm{~kg}$, peut porter une charge de $40 \mathrm{~kg}$ pendant un voyage de 20 jours en parcourant 35 kilomètres par jour.

La production de viande résulte de l'abattage des alpagas et des lamas.

Alpagas et lamas ont un poids élevé à la naissance, semblable dans les deux sexes, dépendant de la nutrition des mères, mais également assez héritable $(0,34 \pm 0,23$ selon Bradford et al 1989). Dans les conditions andines d'élevage, Diaz et al (1991) relèvent des poids moyens à la naissance et à l'âge adulte de 6,2 et $47 \mathrm{~kg}$ (alpagas), 10,5 et $101 \mathrm{~kg}$ (lamas). En Nouvelle-Zélande, selon Davis et al (1991), des alpagas originaires du Chili, élevés sur prairies irriguées, pèsent 7,7 à $8,4 \mathrm{~kg}$ à la naissance, 25 à $28 \mathrm{~kg}$ à 4 mois - poids atteint entre 9 mois et un an dans les Andes selon Cruz et al (1991) et Calle Escobar (1982) - et environ $70 \mathrm{~kg}$ à l'âge adulte.

Le rendement en carcasse des lamas est respectivement de 55 et $52 \%$ pour des mâles et femelles abattus à 108 et $101 \mathrm{~kg}$ (Medina et Bustinza 1991). La viande de lama, qu'elle soit fraîche ou séchée au soleil (charqui) est peu grasse (eau : $69,2 \%$; protéines : $24,8 \%$; grais- se : $3,7 \%$ ) et d'une saveur proche de celle du porc selon Calle Escobar (1982). La viande d'alpaga serait un peu plus grasse $(7 \%)$ et moins riche en protéines (19\%), mais les données comparatives font défaut.

La production de fibres textiles de qualité est, bien entendu, la ressource économiquement la plus intéressante pour les éleveurs de camélidés sud-américains. Comme dans toutes les espèces exploitées pour leur toison, les qualités recherchées sont la longueur, la finesse, la résistance et l'élasticité des fibres, leur aptitude au feutrage, l'absence de poils grossiers, la couleur et l'homogénéité des toisons, leur propreté et leur rendement au lavage. En ce domaine il existe de très grandes différences entre espèces (tableau 1), de la Vigogne au Guanaco, de l'Alpaga au Lama. C'est essentiellement l'Alpaga qui est l'objet de nombreuses études. Au Pérou, en 1980 , la production de fibres commercialisées était de 2456 tonnes pour un effectif de 2385000 têtes représentant $46 \%$ de la population mondiale d'alpagas.

Le poids des toisons varie avec le sexe, respectivement $1,5-1,7$ et $2,5-2,8 \mathrm{~kg}$ pour les femelles et les mâles (Calle Escobar 1982, Davis et al 1991), et l'alimentation $(+0,4$ à $+0,7$ $\mathrm{kg}$ en conditions favorables).

La toison des alpagas est constituée presque uniquement de fibres de protection, fines (16 à $40 \mu$ ), le plus souvent médullées ce qui les rend comparable au jarre des ovins. Elles sont légères, isolantes, et résistantes. La coloration naturelle est extrêmement variée puisque, selon Calle Escobar (1982), quinze classes de coloration sont officiellement définies. Le rendement (lavé à fond) est de $80 \%$, supérieur à celui des moutons (50 à $75 \%$ selon les conditions d'élevage). Les fibres des animaux de race Suri sont longues mais peu frisées, ce qui nuit à leur élasticité ; en race Huacaya, les fibres sont plus courtes mais plus frisées. La nature des écailles cuticulaires confère aux fibres de camélidés une moins bonne aptitude au feutrage que chez les ovins.

La finesse des fibres varie avec l'âge des alpagas et leur régime alimentaire. Ainsi, Calle Escobar (1982), dans un essai comparatif trouve des diamètres de 27,3 et $32,4 \mu$ (adultes), 21,6 et $27,8 \mu$ (tuis ou antenais), 20,9 et $27,7 \mu$ (cria ou jeunes) respectivement chez les témoins et les animaux mieux nourris. En Nouvelle-Zélande, Davis et al (1991) obtiennent des fibres de $28,8 \mu$ (femelles), $30,6 \mu$ (mâles) et 23,6 $\mu$ (tuis) chez des alpagas élevés dans des conditions favorables.

Chez le Lama, il existe deux types de fibres : des poils de garde, relativement grossiers (40 à $150 \mu)$ et un pelage de protection formé de fibres plus fines ( 10 à $40 \mu$ ), le plus souvent médullées. L'utilisation des toisons pour la production artisanale de tapis ou tissages nécessite une séparation des deux types de fibres.

Comme dans toutes les espèces, les productions dépendent de l'état de santé (morbidité, mortalité). Fowler (1989) a passé en revue les données disponibles qui correspondent essen-

\section{La digestibilité des fourrages grossiers pauvres est meilleure chez les camélidés que chez les ovins et caprins mais identique lorsque les fourrages sont de bonne qualité.}


tiellement aux conditions d'exploitation des Andes péruviennes. Dans ce contexte, lamas et alpagas semblent très comparables aux autres herbivores domestiques, avec, en particulier, une sensibilité élevée aux parasites que leur aptitude à brouter très ras ne fait que renforcer. Les atteintes de maladies à virus semblent rares sans qu'il soit possible de dire si c'est dû à une aptitude naturelle ou à une absence de virus dans des milieux isolés. En France, l'étude des risques sanitaires de ces espèces reste à réaliser.

Passant en revue les problèmes de pathologie de la reproduction, Avila Cazorla (1986) souligne l'existence de nombreux cas de stérilité permanente ou temporaire dans les deux sexes. Ils ont pour origine la cryptorchidie et l'hypoplasie testiculaire chez le mâle, l'hypoplasie de l'ovaire et les kystes ovariens chez la femelle.

\section{5 / Comportement social}

Les camélidés sud-américains sauvages ont une structure sociale très organisée (Franklin 1983). Les vigognes vivent par groupes familiaux composés d'un mâle, de plusieurs femelles et de leurs petits. Chaque groupe familial occupe un territoire. A côté des groupes familiaux existent, hors saison sexuelle, des groupes de mâles ou des mâles isolés, ceux-ci ayant ou non un territoire. Les guanacos, plus souples, sédentaires ou migrants, ont également une structure sociale plus variée. Elle comprend, à côté des groupes familiaux, des groupes de femelles et des groupes mixtes (groupes souvent temporaires). L'effectif des groupes est plus variable. Les jeunes restent dans le groupe familial jusqu'à l'âge de un an.

Lamas et alpagas ont également, malgré la domestication, une structure sociale forte et hiérarchisée. Le troupeau au pâturage possède un ou plusieurs guetteurs postés à la périphérie en posture d'éveil ou d'agression à l'approche d'un intrus. Toutefois le groupe n'est pas fermé aux autres espèces domestiques : lamas et moutons pâturent ensemble, chacun gardant son rythme propre d'activité (Segrétain 1988). Dumont (1991), étudiant les relations de proximité entre individus au sein d'un troupeau sur parcours de garrigue, constate, chez les lamas, la constitution de petits groupes de 2 à 5 individus, ce qui n'est pas le cas chez les chèvres. La communication entre individus, notamment dans le cadre de l'établissement de rapports hiérarchiques (dominance-subordination), de la surveillance et la défense du groupe, met en jeu des postures de la tête, des oreilles et de la queue (figure 1), mais également des parades agressives dont la plus connue est le jet de salive ou de contenu ruminal, popularisé par Hergé (1955).

Figure 1. Diverses attitudes corporelles chez le Lama (d'après Franklin 1982).

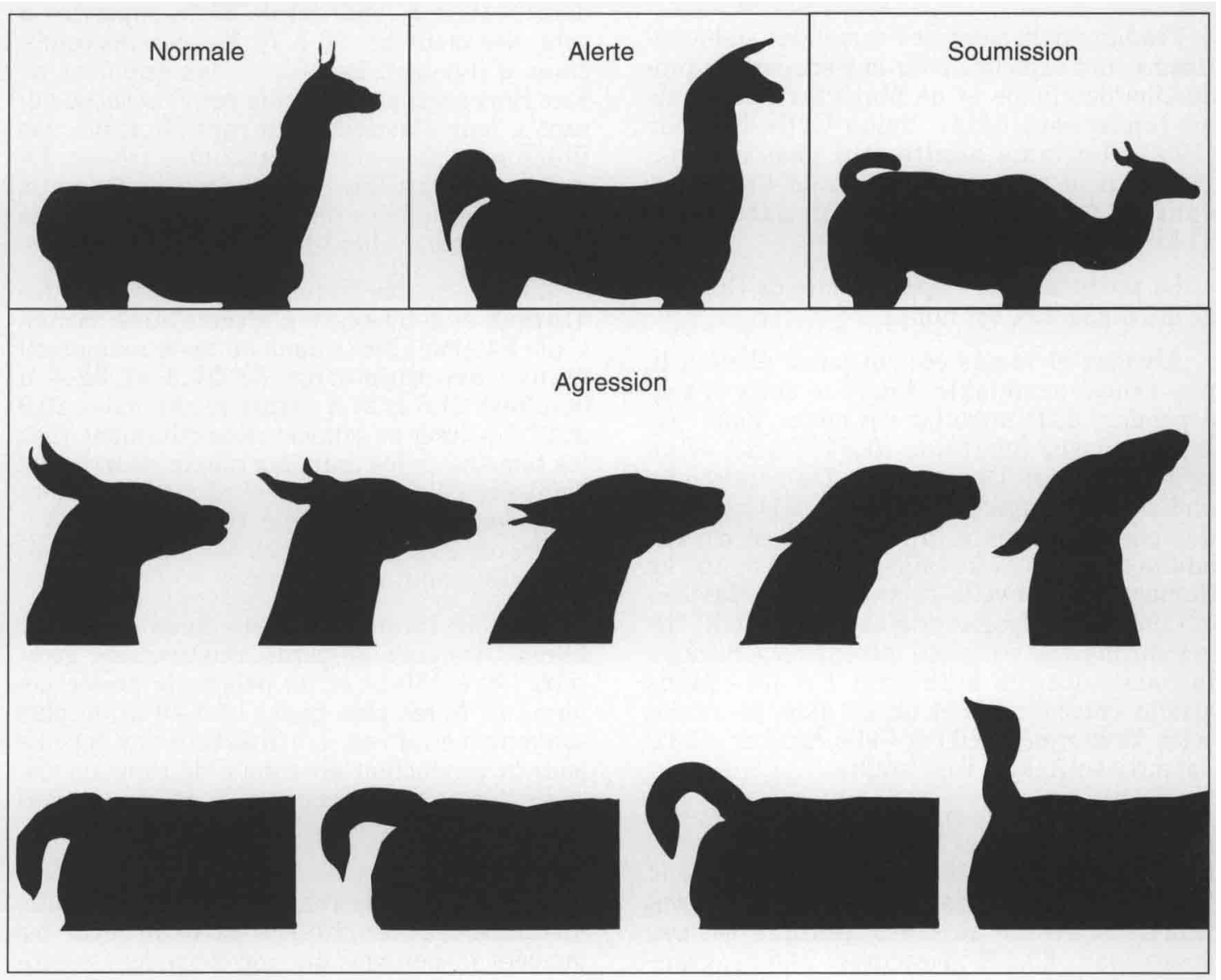




\section{Conclusion}

Au terme de ce survol bibliographique, il faut constater tout d'abord le peu de connaissances disponibles sur l'adaptation et les performances des lamas et, davantage encore, des alpagas, dans les conditions françaises d'élevage. Ceci justifie la poursuite de travaux de recherche si la politique de diversification des productions animales n'exclut pas les camélidés domestiques sud-américains.

Dans leur contexte habituel d'élevage, les alpagas et les lamas ont un mode de reproduction original (accouplements prolongés, ovulation provoquée, nidation tardive dans la corne utérine gauche, mise bas de jour, nouvel accouplement possible peu après la parturition) mais peu productif (faible précocité sexuelle, plus de onze mois de gestation, rareté des naissances multiples, forte mortalité embryonnaire), ce qui limite les possibilités d'expansion rapide de leur élevage.

Alpagas et lamas sont essentiellement des "pâtureurs", les lamas pouvant également consommer, en moindre quantité, des végétations arbustives, notamment lorsque la pelouse herbacée est rasée, et des fourrages grossiers (paille).

A poids métabolique égal, les camélidés sudaméricains ingèrent moins de matière sèche que les ovins et caprins ; ils semblent avoir une meilleure utilisation digestive de la matière sèche avec des régimes très pauvres en protéines.

En dépit d'une bonne sociabilité, ces animaux sont susceptibles de poser quelques problèmes de conduite en raison de la forte hiérarchisation du troupeau et des parades agressives des individus qui en assument la protection.

Sur le plan économique, l'intérêt de l'Alpaga, lié à la qualité de ses fibres, doit être étudié sérieusement avant d'entreprendre des importations hypothétiques et coûteuses. Quant au Lama, malgré sa bonne image médiatique, son intérêt économique reste à prouver.

\section{Références bibliographiques}

Adam C.L., Bourke D.A., Kyle C.E., Young P., Mc Evoy T.G., 1992. Ovulation and embryo recovery in the llama. Proc. 1st Int. Camel Conf., W.R. Allen, A.J. Higgins, I.G. Mayhew, D.H. Snow and J.F. Wade Eds, R \& W Publications Ltd, Newmarket, 125-127.

Adams G.P., Sumar J., Ginther O.J., 1991. Form and function of the corpus luteum in llamas. Anim. Reprod. Sci., 24, 127-138.

Alarcon V., Sumar J., Riera G.S., Foote W.C., 1990. Comparison of three methods of pregnancy diagnosis in alpacas and llamas. Theriogenology, 34, 11191127.

Avila Cazorla E.F., 1986. Physiologie et pathologie de la reproduction de l'alpaga. Mémoire de Maîtrise ès Sciences Vétérinaires, E.N.V. Alfort, France, 47 p.

Bourke D.A., Adam C.L., Kyle C.E., Young P., Mc Evoy T.G., 1992. Superovulation and embryo transfer in the llama. Proc. 1st Int. Camel Conf., W.R. Allen, A.J. Higgins, I.G. Mayhew, D.H. Snow and J.F. Wade Eds, R \& W Publications Ltd, Newmarket, 183-185.

Bradford G.E., Burfening P.J., Cartwright T.C., 1989. Evaluation of production and reproduction of sheep, goat and alpaca genotypes in the small ruminant collaborative research support program. J. Anim. Sci., 67, 3058-3067.

Bravo P.W., 1992. Physiologie de la reproduction chez les camélidés sud-américains. lère Journée technique française sur les Petits Camélidés, Lyon, 4 sept. 1992, 18-28, Association Alpagas et Lamas de France, 05400 Veynes.

Bravo P.W., Fowler M.E., Stabenfeldt G.H., Lasley B.L., 1990. Ovarian follicular dynamics in the llama. Biol. Reprod., 43, 579-585.
Bravo P.W., Stabenfeldt G.H., Fowler M.E., Lasley B.L., 1991. Urinary steroids in the periparturient and postpartum periods through early pregnancy in llamas. Theriogenology, 36, 267-278.

Brunschwig G., 1988. Systèmes d'élevage extensif d'altitude dans les Andes centrales du Pérou. Thèse Doct. Ing., INA-PG., $510 \mathrm{p}$.

Bryant F.C., Farfan R.D., 1984. Dry season forage selection by Alpagas (Lama pacos) in southern Peru. J. Range Manage., 37, 330-333.

Calle Escobar R., 1982. Produccion y mejoramiento de la alpaca. Banco Agrario del Peru, Lima, 334 p.

Carmean B.R., Johnson K.A., Johnson D.E., Johnson L.W., 1992. Maintenance energy requirements of the llama. American J. vet. Res., 53, 1696-1698.

Casamitjana P., 1976. Contribution à l'étude des camélidés sud-américains : leur adaptation aux pâturages des hauts plateaux andins. Thèse Doct. Vét., Toulouse.

Cordesse R., 1992. Utilisation digestive des fourrages par des lamas. Comparaison avec les moutons et les chèvres. 1ère Journée technique française sur les Petits Camélidés, Lyon, 4 sept. 1992, 29-33, Association Alpagas et Lamas de France, 05400 Veynes.

Cordesse R., Inesta M., Gaubert J.L., 1992. Intake and digestibility of four forages by llamas and sheep. Ann. Zootech., 41, 70 (Abstr.).

Cruz C., Bustinza V., Sanchez C., 1991. Indices productivos de alpacas en Chichillapi. VII Conv. Int. Camél. Sudam., San Salvador de Jujuy, 17-20 Abril, Argentina, Resumen, 1.1. 
Davis G.H., Wuliji T., Moore G.H., Bruce G.D., 1991. Growth, reproduction and fibre production of alpacas imported from Chile. Proc. N.Z. Soc. Anim. Prod., 51, $255-258$.

Diaz V., Villalta P., Amachi F., Alencastre R., 1991. La crianza familiar de ovinos alpacas y llamas en la comunidad campesina de Tupac Amaru de Macusani Puno. VII Conv. Int. Camél. Sudam., San Salvador de Jujuy, Argentina, 7-20 Abril, Resumen, 1.5.

De Rouville S., Lapeyronie P., Gousopoulos J., 1991. Rythmes d'activités et choix alimentaires du lama en garrigue languedocienne. 4ème Cong. Int. Terres de Parcours, Montpellier, 22-26 Avril 1991, Abstr. 273.

Dumont B, 1991. Mises au point méthodologiques de l'étude du comportement d'ingestion de chèvres et de lamas sur parcours de garrigue. DAA, ENSA Montpellier. DEA, Univ. Rennes I, $67 \mathrm{p}$.

Engelhardt W.V., Schneider W., 1977. Energy and nitrogen metabolism in the llama. Anim. Res. Dev., $5,68-72$.

Fernandez-Baca S., 1970. Estudios sobre la reproduccion en la alpaca (Lama Pacos). IVITA Bol. extraord., 4, 33-42. Cité par Avila Cazorla, 1986.

Fernandez-Baca S., Calderon W., 1966. Métodos de coleccion de semen de la alpaca. Rev. Fac. Med. Vet. Univ. Nac. May. S. Marcos, Peru, 18-20, 13.

Fernandez-Baca S., Hansel W., Novoa C., 1970. Embryonic mortality in the alpaca. Biol. Reprod., 3, $243-251$.

Fowler M.E., 1989. Medicine and surgery of southamerican camelids : llama, alpaca, vicuna, guanaco. M.E. Fowler Ed., Iowa State Univ. Press, Ames, 391 p.

Franklin W.L., 1982. Llama language. Llamas World, 1, 6-11.

Franklin W.L., 1983. Contrasting socioecologies of South America's wild camelids : the vicuna and the guanaco. In "Advances in the study of mammalian behavior", J.F. Eisenberg and D.G. Kleiman Eds, Special Publication $n^{\circ} 7$, The American Society of Mammalogists, 573-629.

Giudicelli C., 1991. Elever le lama. Comment? Pourquoi ? Crépin-Leblond Ed., 128 p.

Hergé, 1955. Les aventures de Tintin : le temple du soleil. Casterman Ed., 62 p.

Huisa T., 1986. Composicion botanica y valor nutricional de la dieta de alpaca (Lama pacos) en la época de la seca. Tesis B.S. UNSAAC.

Medina G., Bustinza V., 1991. Crecimiento predestete en llamas. VII Conv. Int. Camél. Sudam., San Salvador de Jujuy, Argentina, 7-20 Abril, Resumen, 1.4

Moro M., 1956. Contribucion al estudio de la leche de las alpacas. Revta. Fac. Med. Vet., 7-11, 117-141. Cité par Avila Cazorla, 1986.
Palomino H., Avila E., Tabachi L., Gomez E., 1985. Transferencia de embriones para el mejoramiento de los camélidos sudamericanos. Revta. de Ciencias Veterinarias. 1, 8-11. Cité par Avila Cazorla 1986.

Pollard J.C., Moore G.H., Littlejohn R.P., 1991. The sexual behaviour of alpacas imported to New Zealand from Chile. Proc. N.Z. Soc. Anim. Prod., 51, 43-46.

Reiner R.J., Bryant F.C., 1986. Botanical composition and nutritional quality of alpaca diets in two andean rangeland communities. J. Range Manage., 39, 424-427.

Reiner R.J., Bryant F.C., Farfan R.D., Craddock B.F., 1987. Forage intake of alpacas grazing andean rangeland in Peru. J. Anim. Sci., 64, 868-871.

San Martin F., 1987. Comparative forage selectivity and nutrition of south american camelids and sheep. Ph.D. Dissert., Texas Tech. Univ.

San Martin F., 1991. Nutricion y alimentacion ; Produccion de ruminantes menores : Alpacas. Impresion resumen, Lima, Peru.

San Martin M., Copaira M., Zuniga J., Rodriguez R., Bustinza G., Acosta L., 1968. Aspects of reproduction in the alpaca. J. Reprod. Fert., 16, 395399.

Segrétain V., 1987. L'alpaga, camélidé sudaméricain : notes sur l'animal et sa conduite d'élevage. D.E.S.-U.S.T.L., Montpellier, 39 p.

Segrétain V., 1988. Comportement du lama, seul ou en association avec les ovins, sur prairie irriguée ou parcours de Crau. D.E.A.-U.S.T.L., Montpellier, 50 p.

Segrétain V., Lapeyronie P., Molénat G., Bosc P., 1989. Comportement du lama seul ou en association avec des brebis sur prairies irriguées ou parcours de Crau. XVIth International Grassland Congress, Nice, II, 1275-1276.

Sumar J., Leyva V., 1981. Coleccion de semen mediante la vagina artificial en la alpaca (Lama pacos). Actas IV Conv. Int. Camél. Sudam., Punta Arenas, Corp. Nat. For. Inst. Patagonie, Chile. Cité par Sumar J.et Garcia M., 1986.

Sumar J., Garcia M., 1986. Fisiologia de reproduccion de la alpaca. In "Nuclear and related Techniques in animal Production and Health", Proceedings of an International Symposium, I.A.E.A. - F.A.O., Vienne, Mars 17-21, I.A.E.A.-S.M. 292/17, 149-177.

Vidal-Rioja L., Semorile L., Crisci J., 1991. Genética molecular de camélidos. Analisis de filogenia mediante digestion del ADN con enzimas de restriccion. VII Conv. Int. Camél. Sudam., San Salvador de Jujuy, Argentina, 7-20 Abril, Resumen, 2.16 .

Villca Z., Abasto P., Genin D., 1992. Uso comparativo de la paja brava por llamas y ovinos en el altiplano desértico. Communication personnelle. 


\section{Summary}

\section{Some facts about South American Camelids.}

A literature study to assess the feasibility of raising South American camelids within the concept of land management and diversification of livestock production is carried out.

There are four species of camelids found in South America : two wild species the "Guanaco" and the "Vicuña" and two domestic species the Alpaca and the Llama. They are found on the high Andean plateau. The domestic species can breed throughout the year, autumn being the most favourable period. The breeding system of these animals has a number of special features : the male attains puberty at the age of about 12 months but is rarely capable of breeding before the age of 3 years, being handicapped by preputial adhesions. In the female, ovulation (usually one) is triggered off by copulation. The development of the single foetus always occurs in the left horn of the uterus. Gestation lasts approximately 345 days. Mating can recommence almost immediately. The weight at birth ranges from 7 to $8 \mathrm{~kg}$ for the Alpaca and from 10 to $11 \mathrm{~kg}$ for the Llama. Weaning occurs at the age of 7 to 9 months.

Both the Alpaca and the Llama are grazers, feeding essentially on grass, including very tall and rough tufts, but the llamas are capable of feeding on shrubs when grass is scarce. Generally, the dry matter intake per metabolic weight of alpacas and llamas is lower than that of sheep and goats (30 to $55 \mathrm{~g} \mathrm{DM} / \mathrm{kg} \mathrm{P}^{0.75}$ ). On similar diets, they consume 20 to $40 \%$ less feed than sheep and goats. The rate of utilization of the digestible organic matter and nitrogen is similar in the four species on a good quality diet but is higher in the case of fibrous and low protein diets for alpacas and llamas.

In south America, camelids coexist with small ruminants. In their social structure, they exhibit a hierarchy and some group defensive mechanisms. It is therefore necessary to take certain precautions when handling them.

Alpacas are mainly raised for their fleece which is made up of fine medulated fibers of 25 to $30 \mu$. Total yields are $1.7 \mathrm{~kg}$ for the females and $2.8 \mathrm{~kg}$ for the males with a clean fleece yield of $80 \%$. Llamas have coarser fleece which contains kemp fibers. The fleece can be used in craft work.

In France, no economic survey which can stimulate farmers to raise these animals has been carried out at the time of writing.

PRUD'HON M., CORDESSE R., DE ROUVILLE S., THIMONIER J. 1993. Les camélidés sud-américains : le point des connaissances. INRA Prod. Anim., 6 (1), 5 - 15. 